\author{
Military Technical College \\ Kobry El-Kobbah, \\ Cairo, Egypt
}

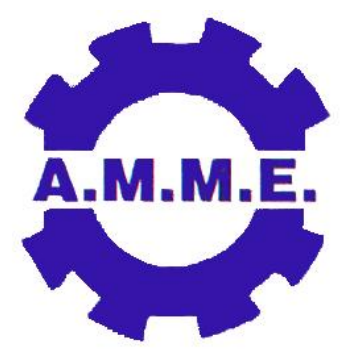

$14^{\text {th }}$ International Conference on Applied Mechanics and Mechanical Engineering.

\title{
Closed-Form Solution For Laminated Bio-composite Plates With Interlayer Slip
}

\author{
By
}

R. Hussein*

\begin{abstract}
:
In the literature, very few publications are available that deal with the structural response of biocomposite plates with interlayer slips. Realistically, the bonding in the plates is rigid enough to make a significant contribution to the overall structural integrity of the plate, yet flexible enough to permit interlayer shear deformation. A closed-form solution for laminated biocomposite plates assembled with non-rigid bonding and subjected to edgewise loading is presented in this paper. The edgewise load can be uniaxial or biaxial; applied to the facings only, core only, or to both components. The solution satisfies the equilibrium equations of the layers, the compatibility equations of stresses and strains at the interfaces, and the boundary conditions. To investigate the effects of the finite values of bonding stiffness on the mechanical responses of the plates, numerical evaluations are conducted.
\end{abstract}

The results obtained have shown that the adhesive stiffness has a strong effect on the plate mechanical response. Beyond a certain level of stiffness, the usual assumption of perfect bonding used in classical theories is acceptable. This could provide an answer to what constitutes perfect bonding in terms of the ratio of the inner layer stiffness to the bonding stiffness.

\section{Keywords:}

Biocomposites - Biodegradable - Bond - Delamination - Interlayer - Plates. 
* Engineering Systems, Cairo, Egypt

\section{Background:}

Significant advances in the development of biodegradable composites have promoted the modern application of biocomposite plates to meet modern challenges $(3,7,11,12,20,22$, 26,27 , and 30 ). They have gained unprecedented popularity in high-performance products that need to be lightweight yet strong enough to take harsh loading conditions. In recent years, increasing attention has turned to novel composites for the resistance to blasts due to possible manmade incidents $(2,21$, and 31$)$. Furthermore, the increased interests in environmental sustainability of resources have propelled considerable advancements in biocomposites made from natural materials. Through natural and synthetic fibers, hybrid designs are emerging as competitive composites for conventional structural components (22, 26 , and 27). They introduce viable alternatives to classic systems and became one of the fastest growing products for decks, bridges, emergency shelters, and off-shore deck platforms.

It should be emphasized that bonding plays, unavoidable role in the manufacturing and performance of composite systems. However, modern challenges urge the development of natural bonding to substitute for the synthetic thermosetting resins in order to decrease the dependence on fossil fuels and meet ever-increasing environmental requirements. An inprogress research $(3$, and 8 ) aims at the potential use of the lignin and a formaldehyde-free bonding.

Existing analytic and experimental methods of analysis of composite have invariably assumed perfect bonding between layers $(1,23,24,21,29$, and 31). Nevertheless, interlayer slips do occur because of the finite bonding stiffness; the bonding creep under sustained loads and environmental effects. Analysis of wood joist floor systems, taking into account interlayer shear stresses, was done by Goodman et al. (4, 5, 6, and 30). The interlaminar shear in composites under plane stress was investigated analytically by Puppo and Evensen (25) and with the finite element method by Isakson and Levy (19). In a series of analytic and experimental studies, the author has investigated the structural behaviors of plates with interlayer slips and under transverse and thermal loads (14, 15, 16, and 18) and beamcolumns with interlayer slip (18).

The objective of this paper is to ascertain the effect of interlayer slips on the performance of plates due to edgewise loads. Because of the inherit complexity of composite plates, this paper uses three layers plates in order to institute the foundation for future further studies. In this way, high confidence is established in the reliability of the very complex mathematic approach of the problem.

\section{Formulation:}

Consider a composite plate of span $2 a$ and width $2 \mathrm{~b}$, subjected to in-plane biaxial loads as shown in Figure 1. The facings or outer layer are of equal thickness $t_{f}$. The middle layer of a thickness $2 t_{c}$, has a modului of elasticity, $E_{c x}$ and $E_{c y}$ usually significantly less than those of 
the faces $E_{f x}$ and $E_{f y}$. However, its shear modului $G_{c x y}, G_{c x z}$ and $G_{c y z}$ should be high enough to develop the interaction required between the layers. The adhesive between the facings and core has finite stiffness $\mathrm{K}_{\mathrm{x}}$ and $\mathrm{K}_{\mathrm{y}}$. This kind of problem has been attacked using the fundamentals of theory of elasticity $(9,10,13,14,15,16,17$, and 18). Generally, the stress state in the faces and core elements is shown in Figure 2. The equilibrium of the face element requires that

$$
\begin{aligned}
& \frac{\partial \sigma_{f x}}{\partial x}+\frac{\partial \tau_{f y x}}{\partial y}-\frac{q_{x}}{t_{f}}=0 \\
& \frac{\partial \sigma_{f y}}{\partial y}+\frac{\partial \tau_{f x y}}{\partial x}-\frac{q_{y}}{t_{f}}=0
\end{aligned}
$$

in which

$$
\begin{array}{ll}
\sigma_{\mathrm{fx}}, \sigma_{\mathrm{fy}} & =\text { Normal stress components in faces; } \\
\tau_{\mathrm{fxy}}, \tau_{\mathrm{fyx}} & =\text { Shear stress components in faces; } \\
\mathrm{q}_{\mathrm{x}} \text { and } \mathrm{q}_{\mathrm{y}} & =\text { Interlayer shear stress; } \\
\mathrm{t}_{\mathrm{f}} & =\text { The thickness of the face; } \\
\mathrm{f} & =\text { Subscript denoting face; } \\
\mathrm{x}, \mathrm{y} & =\text { Coordinate axes. }
\end{array}
$$

The state of stress in the core must satisfy the following equilibrium equations.

$$
\begin{aligned}
& \frac{\partial \sigma_{c x}}{\partial x}+\frac{\partial \tau_{c y x}}{\partial y}+\frac{\partial \tau_{c z x}}{\partial z}=0 \\
& \frac{\partial \sigma_{c y}}{\partial y}+\frac{\partial \tau_{c x y}}{\partial x}+\frac{\partial \tau_{c z y}}{\partial z}=0
\end{aligned}
$$

in which

$\sigma_{\mathrm{cx}}, \sigma_{\mathrm{cy}}, \sigma_{\mathrm{cz}} \quad=$ Normal stress in the core;

$\tau_{\mathrm{cxy}}, \tau_{\mathrm{cyz}}, \tau_{\mathrm{czx}}=$ Shear stress in the core;

$\mathrm{C}=$ Subscript denoting core.

The normal stress components in the facings and core must also satisfy the overall equilibrium equations, which are

$$
\begin{aligned}
& 2 \operatorname{tf} \int_{y=0}^{y=2 b} \sigma_{f x} d y+\int_{y=0}^{y=2 b} \int_{z=-t c}^{z=t c} \sigma_{c x} d y d z+\int_{y=0}^{y=2 b} p_{x} d y=0 \\
& 2 \operatorname{tf}_{y=0}^{y=2 a} \sigma_{\mathrm{fy}} d x+\int_{y=0}^{\mathrm{y}=2 \mathrm{a}} \int_{z=-\mathrm{tc}}^{\mathrm{z}=\mathrm{tc}} \sigma_{\mathrm{cy}} \mathrm{dx} d z+\int_{\mathrm{y}=0}^{\mathrm{y}=2 \mathrm{a}} \mathrm{p}_{\mathrm{y}} \mathrm{dx}=0
\end{aligned}
$$

where $p_{x}$ and $p_{y}$ are the applied edge loads. At the interfaces between the core and the skins, 
the stresses and strains must be compatible, thus

$$
\begin{aligned}
& \mathrm{q}_{\mathrm{x}}=\left.\tau_{\mathrm{czx}}\right|_{\mathrm{Z}= \pm \mathrm{tc}} \\
& \mathrm{q}_{\mathrm{y}}=\left.\tau_{\mathrm{czy}}\right|_{\mathrm{Z}= \pm \mathrm{tc}} \\
& \frac{\partial \Delta_{\mathrm{x}}}{\partial \mathrm{x}}=\varepsilon_{\mathrm{fx}}-\left.\varepsilon_{\mathrm{cx}}\right|_{\mathrm{Z}= \pm \mathrm{t}_{\mathrm{c}}} \\
& \frac{\partial \Delta_{\mathrm{y}}}{\partial \mathrm{y}}=\varepsilon_{\mathrm{fy}}-\left.\varepsilon_{\mathrm{cy}}\right|_{\mathrm{Z}= \pm \mathrm{t}_{\mathrm{c}}} \\
& \gamma_{\mathrm{fxy}}=\left.\gamma_{\mathrm{cxy}}\right|_{\mathrm{Z}= \pm \mathrm{tc}_{\mathrm{c}}}
\end{aligned}
$$

in which

$\varepsilon$ and $\gamma \quad=$ Normal and shear strain, respectively;

$$
\begin{array}{ll}
\Delta_{\mathrm{i}} & =\frac{\mathrm{q}_{\mathrm{i}}}{\mathrm{K}_{\mathrm{i}}} ; \\
\mathrm{K}_{\mathrm{i}} & =\text { Stiffness of adhesive in the i direction. }
\end{array}
$$

Solutions to the problem must also satisfy the prescribed displacement boundary conditions. With respect to a composite plate subjected to edgewise loads, the relevant boundary conditions are

$$
\begin{aligned}
& \text { at } \mathrm{x}=0,2 \mathrm{a} \\
& \sigma_{\mathrm{fx}}=\sigma_{\mathrm{fxo}} \\
& \sigma_{\mathrm{cx}}=\sigma_{\mathrm{cxo}} \\
& \text { at } y=0,2 b \\
& \sigma_{\mathrm{fy}}=\sigma_{\mathrm{fyo}} \\
& \text { at } x=a \\
& \tau_{\mathrm{fxy}}=\tau_{\mathrm{cxy}}=0 \\
& \text { at } y=b \\
& \tau_{\mathrm{fyx}}=\tau_{\mathrm{cyx}}=0 \\
& \sigma_{\mathrm{cy}}=\sigma_{\mathrm{cyo}} \\
& \mathrm{u}_{\mathrm{c}}=\mathrm{u}_{\mathrm{f}}=0 \\
& v_{c}=v_{f}=0 \\
& \sigma_{\mathrm{fxo}} \quad=\mathrm{p}_{\mathrm{fxo}} / \mathrm{t}_{\mathrm{f}} \\
& \sigma_{\mathrm{fyo}} \quad=\mathrm{p}_{\mathrm{fyo}} / \mathrm{t}_{\mathrm{f}} \\
& \mathrm{u} \text { and } \mathrm{v} \quad=\text { displacements in the } \mathrm{x} \text { and } \mathrm{y} \text { directions, respectively. }
\end{aligned}
$$

For the plate in Figure 1, a solution for normal stress components in the core satisfying the boundary conditions in Equations 12 and 13 is considered as $(14,15,16,17,18)$

$$
\begin{aligned}
\sigma_{c x} & =\sum_{\mathrm{m}=1,3, \ldots}^{\infty} \sum_{\mathrm{n}=1,3, . .}^{\infty} \mathrm{A}_{\mathrm{mn}} \phi_{\mathrm{x}} \mathrm{S}_{\mathrm{x}} \mathrm{S}_{\mathrm{y}}+\sigma_{\mathrm{cxo}} \\
\sigma_{\mathrm{cy}} & =\sum_{\mathrm{m}=1,3, . . \mathrm{n}=1,3, . .}^{\infty} \sum_{\mathrm{mn}}^{\infty} \phi_{\mathrm{y}} \mathrm{S}_{\mathrm{x}} \mathrm{S}_{\mathrm{y}}+\sigma_{\text {cyo }}
\end{aligned}
$$

in which

$\sigma_{\mathrm{cxo}}, \sigma_{\mathrm{cyo}} \quad=$ Edge stresses in the core in the $\mathrm{x}$ and $\mathrm{y}$ directions, respectively; $\phi_{\mathrm{x}}=\theta_{\mathrm{x}}\left(2 \theta_{\mathrm{x}} \cosh \theta_{\mathrm{x}} \mathrm{z}+\mathrm{z} \theta_{\mathrm{x}}^{2} \sinh \theta_{\mathrm{x}} \mathrm{z}-\mathrm{t}_{\mathrm{c}} \theta_{\mathrm{x}}^{2} \operatorname{coth} \theta_{\mathrm{x}} \mathrm{t}_{\mathrm{c}} \cosh \theta_{\mathrm{x}} \mathrm{z}\right)-\frac{\alpha_{\mathrm{m}}^{2}}{2} \mathrm{k}_{\phi \mathrm{x}} \cos \alpha_{\phi \mathrm{x}} \mathrm{z}$;

$\phi_{\mathrm{y}}=\theta_{\mathrm{y}}\left(2 \theta_{\mathrm{y}} \cosh \theta_{\mathrm{y}} \mathrm{z}+\mathrm{z} \theta_{\mathrm{y}}^{2} \sinh \theta_{\mathrm{y}} \mathrm{z}-\mathrm{t}_{\mathrm{c}} \theta_{\mathrm{y}}^{2} \operatorname{coth} \theta_{\mathrm{y}} \mathrm{t}_{\mathrm{c}} \cosh \theta_{\mathrm{y}} \mathrm{z}\right)-\frac{\beta_{\mathrm{n}}^{2}}{2} \mathrm{k}_{\phi \mathrm{y}} \cos \alpha_{\phi \mathrm{y}} \mathrm{z} ;$ 
$\mathrm{k}_{\phi x}=-\frac{\theta_{\mathrm{x}} \mathrm{t}_{\mathrm{c}}}{\sinh \theta_{\mathrm{x}} \mathrm{t}_{\mathrm{c}}}+\theta_{\mathrm{x}} \mathrm{t}_{\mathrm{c}} \cosh \theta_{\mathrm{x}} \mathrm{t}_{\mathrm{c}} ;$

$\mathrm{k}_{\phi y}=-\frac{\theta_{\mathrm{y}} \mathrm{t}_{\mathrm{c}}}{\sinh \theta_{\mathrm{y}} \mathrm{t}_{\mathrm{c}}}+\theta_{\mathrm{y}} \mathrm{t}_{\mathrm{c}} \cosh \theta_{\mathrm{y}} \mathrm{t}_{\mathrm{c}} ;$

$\theta_{\mathrm{x}}=\alpha_{\mathrm{m}} \sqrt{\frac{\mathrm{E}_{\mathrm{cx}}}{\mathrm{G}_{\mathrm{cxz}}}} ;$

$\theta_{\mathrm{y}}=\beta_{\mathrm{n}} \sqrt{\frac{\mathrm{E}_{\mathrm{cy}}}{\mathrm{G}_{\mathrm{cyz}}}} ;$

$\alpha_{\phi x} \quad=\frac{m \pi}{2 t_{c}} ;$

$\beta_{\phi y} \quad=\frac{\mathrm{n} \pi}{2 \mathrm{t}_{\mathrm{c}}} ;$

$\mathrm{S}_{\mathrm{x}}, \mathrm{S}_{\mathrm{y}} \quad=\sin \square_{\mathrm{m}} \mathrm{x}$ and $\sin \square_{\mathrm{n}} \mathrm{y}$, respectively;

$\alpha_{\mathrm{m}}, \beta_{\mathrm{n}} \quad=\frac{\mathrm{m} \pi}{2 \mathrm{a}}$ and $\frac{\mathrm{n} \pi}{2 \mathrm{~b}}$, respectively;

$A_{m n}, C_{m n} \quad=\quad$ Unknown coefficients;

$\mathrm{m}, \mathrm{n} \quad=$ Integers.

Using Equations 16 and 17, expressions for the displacement of the core that satisfy the boundary conditions in Equations 14 and 15 are derived as follow

$$
\begin{aligned}
\mathrm{u}_{\mathrm{c}}= & -\frac{1}{\mathrm{E}_{\mathrm{cx}}} \sum_{\mathrm{m}=1,3, . .}^{\infty} \sum_{\mathrm{n}=1,3, . .}^{\infty} \frac{\mathrm{A}_{\mathrm{mn}} \phi_{\mathrm{x}} \mathrm{C}_{\mathrm{x}} \mathrm{S}_{\mathrm{y}}}{\alpha_{\mathrm{m}}}+\frac{v_{\mathrm{cxy}}}{\mathrm{E}_{\mathrm{cy}}} \sum_{\mathrm{m}=1}^{\infty} \sum_{\mathrm{n}=1}^{\infty} \frac{\mathrm{C}_{\mathrm{mn}} \phi_{\mathrm{y}} \mathrm{C}_{\mathrm{x}} \mathrm{S}_{\mathrm{y}}}{\alpha_{\mathrm{m}}}+ \\
& (\mathrm{x}-\mathrm{a})\left(\frac{\sigma_{\text {cxo }}}{\mathrm{E}_{\mathrm{cx}}}-\frac{v_{\mathrm{cxy}} \sigma_{\mathrm{cyo}}}{\mathrm{E}_{\mathrm{cy}}}\right) \\
\mathrm{v}_{\mathrm{c}}= & -\frac{1}{\mathrm{E}_{\mathrm{cy}}} \sum_{\mathrm{m}=1,3, . . \mathrm{n}=1,3, . .}^{\infty} \frac{\mathrm{C}_{\mathrm{mn}} \phi_{\mathrm{y}} \mathrm{S}_{\mathrm{x}} \mathrm{C}_{\mathrm{y}}}{\beta_{\mathrm{n}}}+\frac{v_{\mathrm{cyx}}}{\mathrm{E}_{\mathrm{cx}}} \sum_{\mathrm{m}=1}^{\infty} \sum_{\mathrm{n}=1}^{\infty} \frac{\mathrm{A}_{\mathrm{mn}} \phi_{\mathrm{x}} \mathrm{S}_{\mathrm{x}} \mathrm{C}_{\mathrm{y}}}{\beta_{\mathrm{n}}}+ \\
& (\mathrm{y}-\mathrm{b})\left(\frac{\sigma_{\mathrm{cyo}}}{\mathrm{E}_{\mathrm{cy}}}-\frac{v_{\text {cxy }} \sigma_{\mathrm{cxo}}}{\mathrm{E}_{\mathrm{cx}}}\right)
\end{aligned}
$$

in which
$v \quad=$ Poisson's ration;
$\mathrm{C}_{\mathrm{x}}, \mathrm{C}_{\mathrm{y}} \quad=\cos \square_{\mathrm{m}} \mathrm{x}$ and cos $\square_{\mathrm{n}} \mathrm{y}$, respectively.

The shear strain in the core is obtained by differentiating Eqs. 18 and 19; thus 


$$
\begin{aligned}
& \gamma_{\mathrm{cxy}}= \frac{1}{\mathrm{E}_{\mathrm{cx}}} \sum_{\mathrm{m}=1,3, . .}^{\infty} \sum_{\mathrm{n}=1,3, . .}^{\infty} \mathrm{A}_{\mathrm{mn}} \phi_{\mathrm{x}}\left(-\frac{\beta_{\mathrm{n}}}{\alpha_{\mathrm{m}}}+v_{\mathrm{cyx}} \frac{\alpha_{\mathrm{m}}}{\beta_{\mathrm{n}}}\right) \mathrm{C}_{\mathrm{x}} \mathrm{C}_{\mathrm{y}}+ \\
& \frac{1}{\mathrm{E}_{\mathrm{cy}}} \sum_{\mathrm{m}=1,3, . . \mathrm{n}=1,3, . .}^{\infty} \sum_{\mathrm{mn}}^{\infty} \phi_{\mathrm{y}}\left(-\frac{\alpha_{\mathrm{m}}}{\beta_{\mathrm{n}}}+v_{\mathrm{cxy}} \frac{\beta_{\mathrm{n}}}{\alpha_{\mathrm{m}}}\right) \mathrm{C}_{\mathrm{x}} \mathrm{C}_{\mathrm{y}}
\end{aligned}
$$

By substituting Equations 16, 17 and 20 in Equations 3 and 4, the shear stresses are

$$
\begin{aligned}
& \tau_{\mathrm{cxz}}=\sum_{\mathrm{m}=1,3, . . \mathrm{n}=1,3, . .}^{\infty} \sum_{\mathrm{z}=0}^{\mathrm{z}} \phi_{\mathrm{x}} \mathrm{dz} \mathrm{A}_{\mathrm{mn}}\left[-\alpha_{\mathrm{m}}+\frac{\mathrm{G}_{\mathrm{cxy}}}{\mathrm{E}_{\mathrm{cx}}} \beta_{\mathrm{n}}\left(-\frac{\beta_{\mathrm{n}}}{\alpha_{\mathrm{m}}}+v_{\mathrm{cyx}} \frac{\alpha_{\mathrm{m}}}{\beta_{\mathrm{n}}}\right)\right] \mathrm{C}_{\mathrm{x}} \mathrm{S}_{\mathrm{y}}+ \\
& \frac{\mathrm{G}_{\mathrm{cxy}}}{\mathrm{E}_{\mathrm{cy}}} \sum_{\mathrm{m}=1,3, . .}^{\infty} \sum_{\mathrm{n}=1,3, . .}^{\infty} \int_{\mathrm{z}=0}^{\mathrm{z}} \phi_{\mathrm{y}} \mathrm{dz} \mathrm{C}_{\mathrm{mn}} \beta_{\mathrm{n}}\left(-\frac{\alpha_{\mathrm{m}}}{\beta_{\mathrm{n}}}+v_{\mathrm{cxy}} \frac{\beta_{\mathrm{n}}}{\alpha_{\mathrm{m}}}\right) \mathrm{C}_{\mathrm{x}} \mathrm{S}_{\mathrm{y}} \\
& \tau_{c y z}=\sum_{m=1,3, . .}^{\infty} \sum_{n=1,3, . .}^{\infty} \int_{\mathrm{z}=0}^{\mathrm{z}} \phi_{\mathrm{y}} \mathrm{dz} \mathrm{C}_{\mathrm{mn}}\left[-\beta_{\mathrm{m}}+\frac{\mathrm{G}_{\mathrm{cxy}}}{\mathrm{E}_{\mathrm{cy}}} \alpha_{\mathrm{m}}\left(-\frac{\alpha_{\mathrm{m}}}{\beta_{\mathrm{n}}}+v_{\mathrm{cxy}} \frac{\beta_{\mathrm{n}}}{\alpha_{\mathrm{m}}}\right)\right] \mathrm{S}_{\mathrm{x}} \mathrm{C}_{\mathrm{y}}+ \\
& \frac{\mathrm{G}_{\mathrm{cxy}}}{\mathrm{E}_{\mathrm{cx}}} \sum_{\mathrm{m}=1,3, . . \quad \mathrm{n}=1,3, . .}^{\infty} \int_{\mathrm{z}=0}^{\mathrm{z}} \phi_{\mathrm{x}} \mathrm{dz} \mathrm{A}_{\mathrm{mn}} \alpha_{\mathrm{m}}\left(-\frac{\beta_{\mathrm{n}}}{\alpha_{\mathrm{m}}}+v_{\mathrm{cyx}} \frac{\alpha_{\mathrm{m}}}{\beta_{\mathrm{n}}}\right) \mathrm{S}_{\mathrm{x}} \mathrm{C}_{\mathrm{y}}
\end{aligned}
$$

The interlayer shear stresses are obtained from Equations 21 and 22 as follow

$$
\begin{aligned}
& \mathrm{q}_{\mathrm{x}}=\sum_{\mathrm{m}=1,3, . .}^{\infty} \sum_{\mathrm{n}=1,3, . .}^{\infty}\left(\mathrm{A}_{\mathrm{mn}} \lambda_{\mathrm{gn} 1}+\mathrm{C}_{\mathrm{mn}} \lambda_{\mathrm{gn} 2}\right) \mathrm{C}_{\mathrm{x}} \mathrm{S}_{\mathrm{y}} \\
& \mathrm{q}_{\mathrm{y}}=\sum_{\mathrm{m}=1,3, . . \mathrm{n}=1,3, . .}^{\infty} \sum^{\infty}\left(\mathrm{C}_{\mathrm{mn}} \lambda_{\mathrm{gk} 1}+\mathrm{A}_{\mathrm{mn}} \lambda_{\mathrm{gk} 2}\right) \mathrm{S}_{\mathrm{x}} \mathrm{C}_{\mathrm{y}}
\end{aligned}
$$

in which

$$
\begin{aligned}
& \lambda_{\mathrm{gn} 1}=\left.\int_{\mathrm{z}=0}^{\mathrm{z}} \phi_{\mathrm{x}} \mathrm{dz}\right|_{\mathrm{z}=\mathrm{t}_{\mathrm{c}}}\left[-\alpha_{\mathrm{m}}+\frac{\mathrm{G}_{\mathrm{cxy}}}{\mathrm{E}_{\mathrm{cx}}} \beta_{\mathrm{n}}\left(-\frac{\beta_{\mathrm{n}}}{\alpha_{\mathrm{m}}}+v_{\mathrm{cyx}} \frac{\alpha_{\mathrm{m}}}{\beta_{\mathrm{n}}}\right)\right] \\
& \lambda_{\mathrm{gn} 2}=\left.\frac{\mathrm{G}_{\mathrm{cxy}}}{\mathrm{E}_{\mathrm{cy}}} \int_{\mathrm{z}=0}^{\mathrm{Z}} \phi_{\mathrm{y}} \mathrm{dz}\right|_{\mathrm{z}=\mathrm{t}_{\mathrm{c}}} \beta_{\mathrm{n}}\left(-\frac{\alpha_{\mathrm{m}}}{\beta_{\mathrm{n}}}+v_{\mathrm{cxy}} \frac{\beta_{\mathrm{n}}}{\alpha_{\mathrm{m}}}\right) \\
& \lambda_{\mathrm{gk} 1}=\left.\int_{\mathrm{z}=0}^{\mathrm{z}} \phi_{\mathrm{y}} \mathrm{dz}\right|_{\mathrm{z}=\mathrm{t}_{\mathrm{c}}}\left[-\beta_{\mathrm{n}}+\frac{\mathrm{G}_{\mathrm{cxy}}}{\mathrm{E}_{\mathrm{cy}}} \alpha_{\mathrm{m}}\left(-\frac{\alpha_{\mathrm{m}}}{\beta_{\mathrm{n}}}+v_{\mathrm{cxy}} \frac{\beta_{\mathrm{n}}}{\alpha_{\mathrm{m}}}\right)\right]
\end{aligned}
$$




$$
\lambda_{\mathrm{gk} 2}=\left.\frac{\mathrm{G}_{\mathrm{cxy}}}{\mathrm{E}_{\mathrm{cx}}} \int_{\mathrm{z}=0}^{\mathrm{z}} \phi_{\mathrm{x}} \mathrm{dz}\right|_{\mathrm{Z}=\mathrm{t}_{\mathrm{c}}} \alpha_{\mathrm{m}}\left(-\frac{\beta_{\mathrm{n}}}{\alpha_{\mathrm{m}}}+\nu_{\mathrm{cyx}} \frac{\alpha_{\mathrm{m}}}{\beta_{\mathrm{n}}}\right)
$$

Expressions for the shear stresses in the facings are obtained from Eqs 11 and 20 as

$$
\begin{array}{r}
\tau_{\mathrm{fxy}}=\sum_{\mathrm{m}=1,3, \ldots}^{\infty} \sum_{\mathrm{n}=1,3, \ldots}^{\infty} A_{\mathrm{mn}}\left[\left.\frac{\mathrm{G}_{\mathrm{fxy}}}{\mathrm{E}_{\mathrm{cx}}} \phi_{\mathrm{x}}\right|_{z=\mathrm{tc}}\left(-\frac{\beta_{\mathrm{n}}}{\alpha_{\mathrm{m}}}+v_{\mathrm{cyx}} \frac{\alpha_{\mathrm{m}}}{\beta_{\mathrm{n}}}\right)+\right. \\
\left.\frac{\mathrm{G}_{\mathrm{fxxy}} \beta_{\mathrm{n}}}{\mathrm{K}_{\mathrm{x}}} \lambda_{\mathrm{gn} 1}+\frac{\mathrm{G}_{\mathrm{fxy}} \alpha_{\mathrm{m}}}{\mathrm{K}_{\mathrm{y}}} \lambda_{\mathrm{gk} 2}\right] \mathrm{C}_{\mathrm{x}} \mathrm{C}_{\mathrm{y}}+ \\
\sum_{\mathrm{m}=1,3, \ldots}^{\infty} \sum_{\mathrm{n}=1,3, \ldots}^{\infty} C_{\mathrm{mn}}\left[\left.\frac{\mathrm{G}_{\mathrm{fxy}}}{\mathrm{E}_{\mathrm{cy}}} \phi_{\mathrm{y}}\right|_{z=\mathrm{tc}}\left(-\frac{\alpha_{\mathrm{m}}}{\beta_{\mathrm{n}}}+v_{\mathrm{cxy}} \frac{\beta_{\mathrm{n}}}{\alpha_{\mathrm{m}}}\right)+\right. \\
\left.\frac{\mathrm{G}_{\mathrm{fxx}} \beta_{\mathrm{n}}}{\mathrm{K}_{\mathrm{x}}} \lambda_{\mathrm{gn} 2}+\frac{\mathrm{G}_{\mathrm{fxy}} \alpha_{\mathrm{m}}}{\mathrm{K}_{\mathrm{y}}} \lambda_{\mathrm{gk} 1}\right] \mathrm{C}_{\mathrm{x}} \mathrm{C}_{\mathrm{y}}
\end{array}
$$

An expression for the normal stress in the facings is obtained by from Eqs. 23, 25 and 1;

$$
\sigma_{\mathrm{fx}}=\sum_{\mathrm{m}=1,3, . . \mathrm{n}=1,3, . .}^{\infty}\left(\mathrm{A}_{\mathrm{mn}} \lambda_{\mathrm{z} 1}+\mathrm{C}_{\mathrm{mn}} \lambda_{\mathrm{z} 2}\right) \mathrm{S}_{\mathrm{x}} \mathrm{S}_{\mathrm{y}}+\mathrm{f}(\mathrm{x}, \mathrm{y})
$$

in which

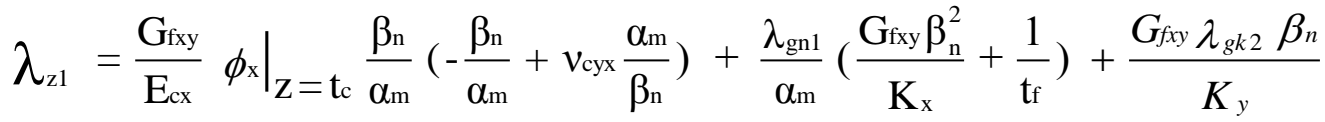

$$
\begin{aligned}
& \lambda_{z 2}=\left.\frac{\mathrm{G}_{\mathrm{fxy}}}{\mathrm{E}_{\mathrm{cy}}} \phi_{\mathrm{y}}\right|_{\mathrm{Z}=\mathrm{t}_{\mathrm{c}}} \frac{\beta_{\mathrm{n}}}{\alpha_{\mathrm{m}}}\left(-\frac{\alpha_{\mathrm{m}}}{\beta_{\mathrm{n}}}+v_{\mathrm{cxy}} \frac{\beta_{\mathrm{n}}}{\alpha_{\mathrm{m}}}\right)+\frac{\lambda_{\mathrm{gn} 2}}{\alpha_{\mathrm{m}}}\left(\frac{\mathrm{G}_{\mathrm{f} x \mathrm{y}} \beta_{\mathrm{n}}^{2}}{\mathrm{~K}_{\mathrm{x}}}+\frac{1}{\mathrm{t}_{\mathrm{f}}}\right)+\frac{\mathrm{G}_{\mathrm{fxx}} \lambda_{\mathrm{gk} 1} \beta_{\mathrm{n}}}{\mathrm{K}_{\mathrm{y}}} \\
& f(x, y)=A \text { function representing the constant of integration. }
\end{aligned}
$$

The function $f(x, y)$ is obtained by using Eqs. $5 ; 16$, and 26 , thus

$$
\sigma_{\mathrm{fx}}=\sum_{\mathrm{m}=1,3, . . \mathrm{n}=1,3, . .}^{\infty}\left[\mathrm{A}_{\mathrm{mn}} \lambda_{\mathrm{z} 1}^{\prime}+\mathrm{C}_{\mathrm{mn}} \lambda_{\mathrm{z} 2}^{\prime}\right] \mathrm{S}_{\mathrm{x}} \mathrm{S}_{\mathrm{y}}+\left(\sigma_{\mathrm{xo}}+\sigma_{\mathrm{cxo}} \frac{\mathrm{t}_{\mathrm{c}}}{\mathrm{t}_{\mathrm{f}}} \sum_{\mathrm{m}=1}^{\infty} \sum_{\mathrm{n}=1}^{\infty} \frac{2}{\mathrm{a} \alpha_{\mathrm{m}}} \frac{2}{\mathrm{~b} \beta_{\mathrm{n}}} \mathrm{S}_{\mathrm{x}} \mathrm{S}_{\mathrm{y}}\right.
$$

in which

$$
\begin{aligned}
& \lambda_{z 1}^{\prime}=\lambda_{z 1}\left(1-\frac{2}{b^{2} \beta_{\mathrm{n}}^{2}}\right)-\frac{2 \int_{0}^{\mathrm{tc}} \phi_{\mathrm{x}} \mathrm{dz}}{\mathrm{tf}^{2} \mathrm{~b}_{\mathrm{n}}^{2}} \\
& \lambda_{\mathrm{z} 2}^{\prime}=\lambda_{\mathrm{z} 2}\left(1-\frac{2}{\mathrm{~b}^{2} \beta_{\mathrm{n}}^{2}}\right)
\end{aligned}
$$

Similarly, an expression for the normal stress is obtained from Eqs. 2, 6, 17 and 24 as

$$
\sigma_{\mathrm{fy}}=\sum_{\mathrm{m}=1,3, . . \mathrm{n}=1,3, . .}^{\infty}\left[\mathrm{A}_{\mathrm{mn}} \lambda_{z 3}^{\prime}+\mathrm{C}_{\mathrm{mn}} \lambda_{z 4}^{\prime}\right] \mathrm{S}_{\mathrm{x}} \mathrm{S}_{\mathrm{y}}+\left(\sigma_{\mathrm{yo}}+\sigma_{\mathrm{cyo}} \frac{\mathrm{t}_{\mathrm{c}}}{\mathrm{tf}_{\mathrm{f}}}\right) \sum_{\mathrm{m}=1}^{\infty} \sum_{\mathrm{n}=1}^{\infty} \frac{2}{\mathrm{a} \alpha_{\mathrm{m}}} \frac{2}{\mathrm{~b} \beta_{\mathrm{n}}} \mathrm{S}_{\mathrm{x}} \mathrm{S}_{\mathrm{y}}
$$

in which 


$$
\begin{aligned}
& \lambda_{z 3}^{\prime}=\lambda_{z 3}\left(1-\frac{2}{\mathrm{a}^{2} \alpha_{\mathrm{m}}^{2}}\right) \\
& \lambda_{z 4}^{\prime}=\lambda_{z 4}\left(1-\frac{2}{\mathrm{a}^{2} \alpha_{\mathrm{n}}^{2}}\right)-\frac{2 \int_{0}^{\mathrm{t}_{\mathrm{c}}} \phi_{\mathrm{y}} \mathrm{dz}}{\mathrm{t}_{\mathrm{f}} \mathrm{a}^{2} \alpha_{\mathrm{m}}^{2}} \\
& \lambda_{\mathrm{z} 3}=\left.\frac{\mathrm{G}_{\mathrm{fxy}}}{\mathrm{E}_{\mathrm{cx}}} \phi_{\mathrm{x}}\right|_{\mathrm{Z}=\mathrm{t}_{\mathrm{c}}} \frac{\alpha_{\mathrm{m}}}{\beta_{\mathrm{n}}}\left(-\frac{\beta_{\mathrm{n}}}{\alpha_{\mathrm{m}}}+\nu_{\mathrm{cyx}} \frac{\alpha_{\mathrm{m}}}{\beta_{\mathrm{n}}}\right)+\frac{\lambda_{\mathrm{gk} 2}}{\beta_{\mathrm{n}}}\left(\frac{\mathrm{G}_{\mathrm{fxy}} \alpha_{\mathrm{m}}^{2}}{\mathrm{~K}_{\mathrm{y}}}+\frac{1}{\mathrm{t}_{\mathrm{f}}}\right)+\frac{\mathrm{G}_{\mathrm{fxy}} \lambda_{\mathrm{gn} 1} \alpha_{\mathrm{m}}}{\mathrm{K}_{\mathrm{x}}} \\
& \lambda_{\mathrm{z} 4}=\left.\frac{\mathrm{G}_{\mathrm{fxy}}}{\mathrm{E}_{\mathrm{cy}}} \phi_{\mathrm{y}}\right|_{\mathrm{Z}=\mathrm{t}_{\mathrm{c}}} \frac{\alpha_{\mathrm{m}}}{\beta_{\mathrm{n}}}\left(-\frac{\alpha_{\mathrm{m}}}{\beta_{\mathrm{n}}}+\nu_{\mathrm{cxy}} \frac{\beta_{\mathrm{n}}}{\alpha_{\mathrm{m}}}\right)+\frac{\lambda_{\mathrm{gk} 1} 1}{\beta_{\mathrm{n}}}\left(\frac{\mathrm{G}_{\mathrm{fxy}} \alpha_{\mathrm{n}}^{2}}{\mathrm{~K}_{\mathrm{y}}}+\frac{1}{\mathrm{t}_{\mathrm{f}}}\right)+\frac{\mathrm{G}_{\mathrm{fxy}} \lambda_{\mathrm{gn} 2} \alpha_{\mathrm{m}}}{\mathrm{K}_{\mathrm{x}}}
\end{aligned}
$$

Finally, the coefficients $A_{m n}$ and $C_{m n}$ can be determined using Eqs. 9 and 10 as

$$
\begin{array}{r}
\mathrm{A}_{\mathrm{mn}}=\frac{\frac{\lambda_{\mathrm{y} 3}}{\lambda_{\mathrm{y} 2}}-\frac{\lambda_{\mathrm{y} 6}}{\lambda_{\mathrm{y} 5}}}{\frac{\lambda_{\mathrm{y} 1}}{\lambda_{\mathrm{y} 2}}-\frac{\lambda_{\mathrm{y} 4}}{\lambda_{\mathrm{y} 5}}} \\
\mathrm{C}_{\mathrm{mn}}=\frac{\frac{\lambda_{\mathrm{y} 3}}{\lambda_{\mathrm{y} 1}}-\frac{\lambda_{\mathrm{y} 6}}{\lambda_{\mathrm{y} 4}}}{\frac{\lambda_{\mathrm{y} 2}}{\lambda_{\mathrm{y} 1}}-\frac{\lambda_{\mathrm{y} 5}}{\lambda_{\mathrm{y} 4}}}
\end{array}
$$

in which

$$
\begin{aligned}
& \lambda_{\mathrm{y} 1}=\frac{\lambda_{\mathrm{zl}}^{\prime}}{\mathrm{E}_{\mathrm{fx}}}-\frac{v_{\mathrm{fxy}} \lambda_{\mathrm{z} 3}^{\prime}}{\mathrm{E}_{\mathrm{fy}}}-\frac{\left.\phi_{\mathrm{x}}\right|_{z=\mathrm{tc}}}{\mathrm{E}_{\mathrm{cx}}}+\frac{\alpha_{\mathrm{m}} \lambda_{\mathrm{gn} 1}}{\mathrm{~K}_{\mathrm{x}}} \\
& \lambda_{y 2}=\frac{\lambda_{z 2}^{\prime}}{E_{\mathrm{fx}}}-\frac{v_{\mathrm{fxy}} \lambda_{\mathrm{z} 4}^{\prime}}{\mathrm{E}_{\mathrm{fy}}}+\frac{\left.v_{\mathrm{cxy}} \phi_{\mathrm{y}}\right|_{\mathrm{z}=\mathrm{tc}}}{\mathrm{E}_{\mathrm{cy}}}+\frac{\alpha_{\mathrm{m}} \lambda_{\mathrm{gn} 2}}{\mathrm{~K}_{\mathrm{x}}} \\
& \lambda_{y 3}=\frac{2}{a \alpha_{m}} \frac{2}{b \beta_{n}}\left[\frac{\sigma_{x o}+\sigma_{c x o} \frac{t_{c}}{t_{f}}}{E_{f x}}-v_{f x y} \frac{\sigma_{y o}+\sigma_{c y o} \frac{t_{c}}{t_{f}}}{E_{f y}}+\frac{\sigma_{c x o}}{E_{c x}}+v_{c x y} \frac{\sigma_{c y o}}{E_{c y}}\right] \\
& \lambda_{\mathrm{y} 4}=\frac{\lambda_{\mathrm{zz}}^{\prime}}{\mathrm{E}_{\mathrm{fy}}}-\frac{\nu_{\mathrm{fyx}} \lambda_{\mathrm{zl}}^{\prime}}{\mathrm{E}_{\mathrm{fx}}}+\frac{\left.\nu_{\mathrm{cyx}} \phi_{\mathrm{x}}\right|_{\mathrm{z}=\mathrm{tc}}}{\mathrm{E}_{\mathrm{cx}}}+\frac{\beta_{\mathrm{n}} \lambda_{\mathrm{gk} 2}}{\mathrm{~K}_{\mathrm{y}}} \\
& \lambda_{\mathrm{y} 5}=\frac{\lambda_{z 4}^{\prime}}{\mathrm{E}_{\mathrm{fy}}}-\frac{v_{\mathrm{fyx}} \lambda_{\mathrm{z} 2}^{\prime}}{\mathrm{E}_{\mathrm{fx}}}-\frac{\left.\phi_{\mathrm{y}}\right|_{\mathrm{z}=\mathrm{tc}}}{\mathrm{E}_{\mathrm{cy}}}+\frac{\beta_{\mathrm{n}} \lambda_{\mathrm{gk} 1}}{\mathrm{~K}_{\mathrm{y}}} \\
& \lambda_{y 6}=\frac{2}{\mathrm{a} \alpha_{\mathrm{m}}} \frac{2}{\mathrm{~b} \beta_{\mathrm{n}}}\left[\frac{\sigma_{\mathrm{yo}}+\sigma_{\mathrm{cyo}} \frac{\mathrm{t}_{\mathrm{c}}}{\mathrm{t}_{\mathrm{f}}}}{\mathrm{E}_{\mathrm{fy}}}-v_{\mathrm{fyx}} \frac{\sigma_{\mathrm{xo}}+\sigma_{\mathrm{cxo}} \frac{\mathrm{t}_{\mathrm{c}}}{\mathrm{t}_{\mathrm{f}}}}{\mathrm{E}_{\mathrm{fx}}}+\frac{\sigma_{\mathrm{cyo}}}{\mathrm{E}_{\mathrm{cy}}}+v_{\mathrm{cyx}} \frac{\sigma_{\mathrm{cxo}}}{\mathrm{E}_{\mathrm{cx}}}\right]
\end{aligned}
$$

\section{Examples}

To demonstrate the effects of bonding, a square plate is considered with general properties 
used in order to represent a wide range of biocomposite components.

$\begin{array}{ll}\mathrm{a}=\mathrm{b} & =20 \mathrm{in} .(1219.2 \mathrm{~mm}) \\ \mathrm{t}_{\mathrm{f}} & =0.04 \mathrm{in} .(1.016 \mathrm{~mm}) ; \\ \mathrm{E}_{\mathrm{fx}}=\mathrm{E}_{\mathrm{fy}} & =10^{7} \mathrm{psi}(68.9 \mathrm{GPa}) ; \\ v_{\mathrm{fxy}}=v_{\mathrm{fyx}} & =0.33 \\ \mathrm{t}_{\mathrm{c}} & =1.0 \mathrm{in} .(50.8 \mathrm{~mm}) ; \\ \mathrm{E}_{c \mathrm{cx}}=\mathrm{E}_{\mathrm{cy}} & =2 \times 10^{6} \mathrm{psi}(137.8 \mathrm{MPa}) \\ \mathrm{G}_{\mathrm{cxy}}=\mathrm{G}_{\mathrm{cxz}}=\mathrm{G}_{\mathrm{cyz}} & =10^{4} \mathrm{psi}(68.9 \mathrm{MPa}) \\ v_{c x y}=v_{c y x} & =0.20\end{array}$

Two loading cases are considered. In the first case a biaxial uniformly distributed stress of intensity $\sigma_{\mathrm{fxo}}=\sigma_{\mathrm{fyo}}=208.3 \mathrm{psi}$ is used. In the second case a uniaxial uniformly distributed stress of intensity $\sigma_{\mathrm{fxo}}=208.3 \mathrm{psi}$ is applied. In each loading case, the load is applied independently first to the face and core, and then concurrently to face and core as shown in Figures 3, 4 and 5. The normal stress in the facings at the plate center and the shear stress in the facings at the plate corner are shown in Figures 6 to 11. It is seen that the face normal stress shows greater sensitivity to the variation of bond stiffness value when the latter is in the lower range; and beyond a certain level of stiffness, the adhesive can be practically considered as rigid. A change in $\mathrm{K}_{\mathrm{x}}$ (or $\mathrm{K}_{\mathrm{y}}$ ) value for example from $10^{3}$ to $2 \times 10^{3} \mathrm{psi} / \mathrm{in}$ induces a stress decrease almost 6 times in the uniaxial case and 5 times in the biaxial case greater than when $\mathrm{K}_{\mathrm{x}}$ (or $\mathrm{K}_{\mathrm{y}}$ ) changes from $9 \times 10^{3}-10^{4} \mathrm{psi} / \mathrm{in}$. The changes are 24 and 27 due to uniaxial core and combined edge loads, respectively, 32 and 22 due to biaxial core and combined edge loads, respectively. In all load cases, the face shear stress is practically independent of bonding stiffness. Unlike the mechanical behavior of other plates with nonrigid adhesive $(9,10,11,12,13,14,15,16$ and 18), this study reveals that interlayer shears are insignificant. This is due to the absence of transverse loads which induce high transverse shear forces. This analysis has yet to bring up an important point. By using existing theories $(1,24)$, stress components in composite plates may be determined only at high values of bond stiffness with a small margin of error, otherwise the $K$ values must be included in the analysis. Another important point has yet to come out of this analysis. By common sense, it can be felt that a very stiff adhesive would be unnecessary if the core was too soft, and the converse would be unwise. This is quantitatively shown in Figures 6 to 11 which show that the ratio of core stiffness to bonding stiffness is one of the main parameters influencing the behavior of composite plates.

\section{Conclusions:}

An analysis of orthotropic composite plates taking into account the effects of the finite bonding stiffness has been presented in this paper. The edgewise load can be uniaxial or biaxial; applied to the facings only, core only, or to both components. The solution satisfies the equilibrium equations of the face and core, the compatibility equations of stresses and strains at the interfaces, and the boundary conditions. The numerical results have shown that the bonding stiffness, up to a certain level, has a strong effect on the plate response. Beyond this level, the usual assumption of perfect bonding in the literature is quite acceptable. The answer to what constitute perfect bonding may be best answered in terms of the ratio of core stiffness to the bond stiffness, rather than on the individual constituent material. 


\section{References:}

1. Allen, H. G. (1969). Analysis and Design of Structural Sandwich Panels. Pergamon Press Ltd.

2. Andrews, E. W. and Moussa, N. A. (2009). Failure mode maps for composite sandwich panels subjected to air blast loading. International Journal of Impact Engineering, Vol. 36.

3. Bujanovic, B., Personal conversation, 2009.

4. Goodman, J. R., and Popov, E. P. (1968). Layered Beam Systems with Interlayer Slip. Journal of the Structural Engineering Division, ASCE, Vol. 94, No. ST11.

5. Goodman, J. R. (1969). Layered Wood Systems with Interlayer Slip. Wood Science, Vol. 1, No. 3.

6. Goodman, J. R., Vanderbilt, M. D., Criswell, M. E., and Bodig, J. (1974). Composite and Two-Way Action in Wood Joist Floor Systems," Wood Science, Vol. 7, No. 1.

7. Haan, D. L. (1996). A New Foam Core for Demanding Sandwich Structures. Proceedings of the Third International Conference on Sandwich Construction, Southampton UK.

8. Huang, J. and Li, K. (2008). A New Soy Flour-Based Adhesive for Making Interior Type II Plywood. Journal of the American Oil Chemists' Society, Vol. 85, No. 1.

9. Hussein, R. (2002). Exact Closed-Form Solutions for Fiber-Fiber and Bond-Fiber Interactions with Imperfect Bonding. Progress in Paper Physics Conference, Skaneateles NY.

10. Hussein, R. (2002). An Analytic Solution for Fiber-Bond Interactions. The $116^{\text {th }}$ ESPRA Conference, Betlehem PA.

11. Hussein, R. and Cheremisinoff, N. P. (1993). Polymer Applications in the Construction Industry. Chapter 26, Elasomer Technology Handbook, CRC Press.

12. Hussein, R. (1993). Polymers in Sandwich Construction. Chapter 27, Elasomer Technology Handbook, CRC Press.

13. Hussein, R.(1992). Effects of Bonding Stiffness on Thermal Stresses in Sandwich Panels. Journal of the Aerospace Engineering Division, ASCE, Vol. 5, No. 4.

14. Hussein, R. et al (1989). Thermal Stresses in Sandwich Panels With Interlayer Slips. Journal of Thermal Stresses, Vol. 12, No. 2.

15. Hussein, R. (1986). Composite Panels/Plates - Analysis and Design. Technomic Publishing Inc.

16. Hussein, R. (1984). Sandwich Plates With Interlayer slips. Journal of the Engineering Mechanics Division, ASCE, Vol. 110, No. EM4.

17. Hussein, R. (1984). Thermal Stresses in Sandwich Panels," Canadian Aeronautics and Space Journal, Vol. 30, No. 2.

18. Hussein, R. et al (1982). Sandwich Beam-Columns With Interlayer slips. Journal of the Engineering Mechanics Division, ASCE, Vol. 108, No. EM2.

19. Isakson, G. and Levy, A. (1971). Finite Element Analysis of Interlaminar Shear in Fibrous Composites. Journal of Composite Materials, Vol. 5.

20. Karbhari, V. M. (1997). Application of Composite Materials to the Renewal of Twenty-First Century Infrastructure. Proceedings of the Eleventh Conference on Composite Materials," Melbourne Australia.

21. Kepler, J.A. and Hansen, M. R. (2007). Numerical Modeling of Sandwich Panel Response to Ballistic Loading - Energy Balance for Varying Impactor Geometries. No. 9.

22. Mohanty, A. et. al. (2005). Natural Fibers, Biopolymers, and Biocomposites. CRC Press, Florida. 
23. Noor, A. K. et al. (1996). Computational Models for Sandwich Panels and Shells. Applied Mechanics Reviews, Vol. 49, No. 3.

24. Plantema, F. J. (1966). Sandwich Construction - The Bending and Buckling of Sandwich Beams, Plates and Shells. John Wiley and Sons, Inc.

25. Puppo, A. H., and Evensen, H. A. (1970). Interlaminar Shear in Laminated Composites under Generalized Plane Stress," Journal of Composite Materials, Vol. 4.

26. Rigoberto, B. et. al. (2005). Sustainable Cellular Biocomposites from Natural Fibers and Unsaturated Polyester Resin for Housing Panel Applications. Journal of Polymers and the Environment, Vol. 13, No. 2.

27. Satyanarayana, K. G. (2007). Biodegradable Composites based on lignocellulosic Fibers. International Conference on Advanced Materials and Composites (ICAMC).

28. Swanson, S. R. (1997). Introduction to Design and Analysis with advanced Composite Materials, Prentice-Hall Publisher.

29. The university of Maine, http://www.aewc.umaine.edu/.

30. Vanderbilt, M. D., Goodman, J. R., and Criswell, M. E. (1974). Service and Overload Behavior of Wood Joist Floor Systems. Journal of the Structural Engineering Division, ASCE, Vol. 100, No. ST1.

31.Zhu, F., et. al. (2009). A numerical simulation of the blast impact of square metallic sandwich panels," International Journal of Impact Engineering, Vol. 36.
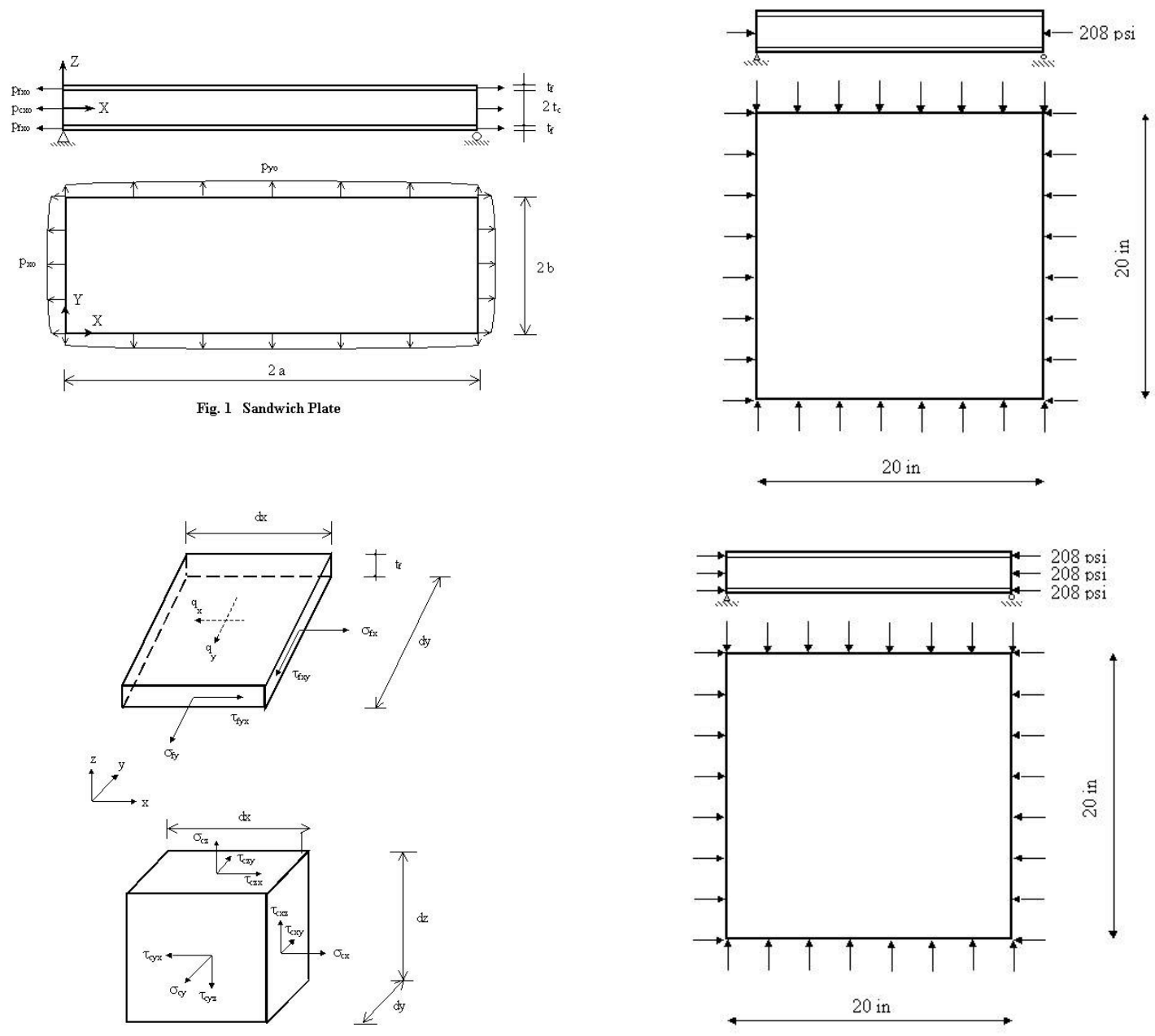

Fig. 2 Stress State in Sandwich Element 

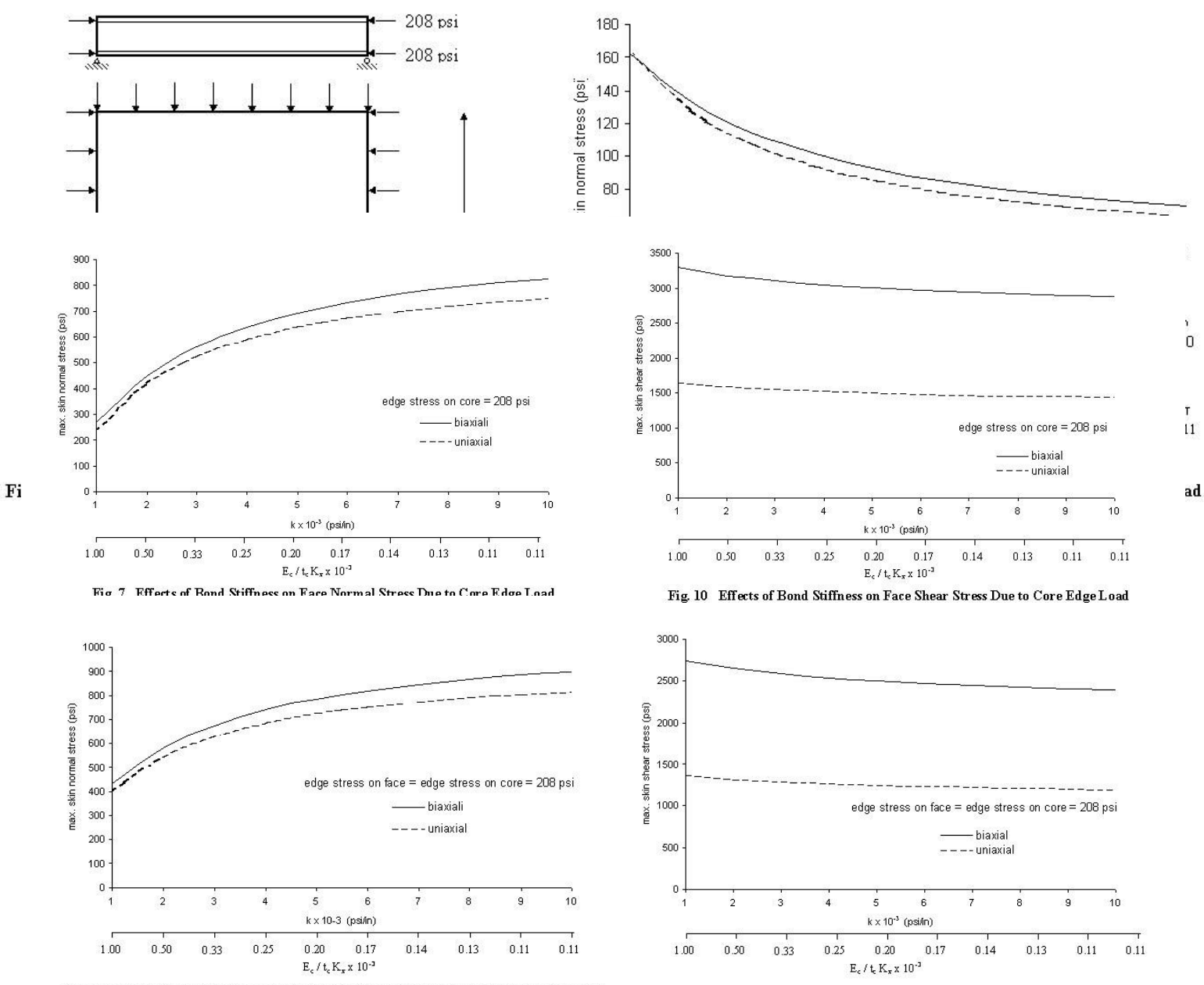

Fig. 8 Effects of Bond Stiffness on Face Normal Stress Due to Face and Core Edge Load

Fig. 11 Effects of Bond Stiffness on Face Shear Stress Due to Core Edge Load

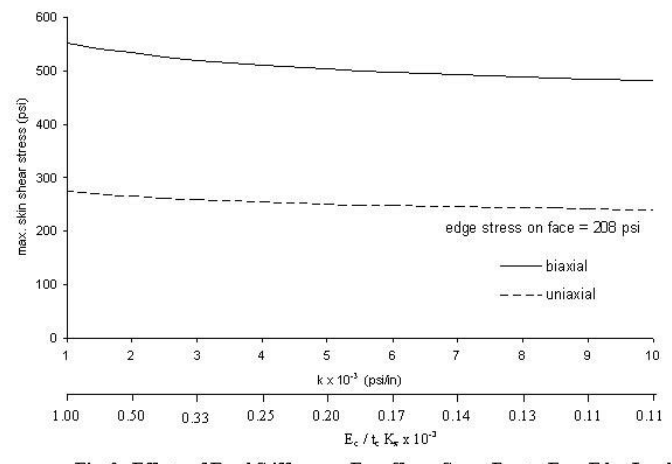

Fig. 9 Effects of Bond Stiffness on Face Shear Stress Due to Face Edge Load 